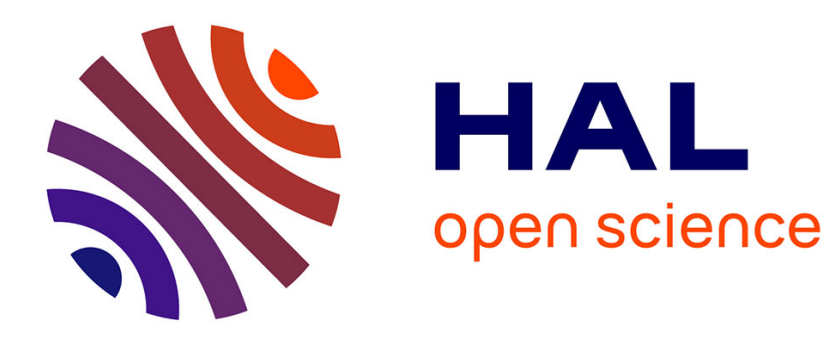

\title{
On a shape derivative formula in the Brunn-Minkowski theory
}

A Boulkhemair

\section{To cite this version:}

A Boulkhemair. On a shape derivative formula in the Brunn-Minkowski theory. 2015. hal-01140162

\author{
HAL Id: hal-01140162 \\ https://hal.science/hal-01140162
}

Preprint submitted on 20 May 2015

HAL is a multi-disciplinary open access archive for the deposit and dissemination of scientific research documents, whether they are published or not. The documents may come from teaching and research institutions in France or abroad, or from public or private research centers.
L'archive ouverte pluridisciplinaire HAL, est destinée au dépôt et à la diffusion de documents scientifiques de niveau recherche, publiés ou non, émanant des établissements d'enseignement et de recherche français ou étrangers, des laboratoires publics ou privés. 


\title{
On a shape derivative formula in the Brunn-Minkowski theory
}

\author{
A. Boulkhemair \\ Laboratoire de Mathématiques Jean Leray, \\ CNRS UMR6629/ Université de Nantes, \\ 2, rue de la Houssinière, BP 92208, 44322 Nantes, France. \\ e-mail : boulkhemair-a arobase univ-nantes.fr
}

\begin{abstract}
We extend a formula for the computation of the shape derivative of an integral cost functional with respect to a class of convex domains, using the so called support functions and gauge functions to express it. This is a priori a formula in shape optimization theory. However, the result also happens to be an extension of a well known formula from the Brunn-Minkowski theory of convex bodies.
\end{abstract}

\section{Introduction}

This work is a continuation of that undertaken in [2]. There, motivated by numerical applications in shape optimization, we proved a formula for the computation of the shape derivative for an integral cost functional with respect to a class of convex domains, using what is called support functions in convex analysis to express such a derivative. To be more precise, let us define our set of admissible domains by

$$
\mathcal{O}=\left\{\Omega \subset \mathbb{R}^{n} ; \Omega \text { is open, non empty, bounded and convex }\right\}
$$

and let us consider the functional $J$ defined on $\mathcal{O}$ by

$$
J(\Omega)=\int_{\Omega} f d x
$$

where $f$ is a fixed function defined in $\mathbb{R}^{n}$. In [2], we proved the following : 
Theorem If $\Omega_{0}, \Omega \in \mathcal{O}, \Omega_{0}$ is of class $C^{2}$ and the function $f$ is in the Sobolev space $W_{\text {loc }}^{1,1}\left(\mathbb{R}^{n}\right)$, that is, $f$ is a locally integrable function whose distributional (or weak) partial derivatives are also locally integrable in $\mathbb{R}^{n}$, then, we have the following limits

$$
\begin{gathered}
\lim _{\varepsilon \rightarrow 0^{+}} \frac{J\left((1-\varepsilon) \Omega_{0}+\varepsilon \Omega\right)-J\left(\Omega_{0}\right)}{\varepsilon}=\int_{\partial \Omega_{0}} f(x)\left(P_{\Omega}\left(\nu_{0}(x)\right)-P_{\Omega_{0}}\left(\nu_{0}(x)\right)\right) d \sigma(x), \\
\lim _{\varepsilon \rightarrow 0^{+}} \frac{J\left(\Omega_{0}+\varepsilon \Omega\right)-J\left(\Omega_{0}\right)}{\varepsilon}=\int_{\partial \Omega_{0}} f(x) P_{\Omega}\left(\nu_{0}(x)\right) d \sigma(x),
\end{gathered}
$$

where $\nu_{0}(x)$ denotes the exterior unit normal vector to $\partial \Omega_{0}$ at $x$, and $P_{\Omega_{0}}, P_{\Omega}$ are the support functions of the domains $\Omega_{0}, \Omega$, respectively.

See Section 2 for the notion of support function of a convex set. This result was an extension of a similar one proved by A. Niftiyev and Y. Gasimov in [1] under the assumptions that $\Omega_{0}, \Omega \in \mathcal{O}$ are of class $C^{2}$ and the function $f$ is of class $C^{1}$, and where only the first limit was considered. The theorem gives formulas for the computation of the shape derivative of $J$ with respect to convex domains, using the support functions of the domains to express it, and our interest in such formulas came first from numerical considerations as we already said. In fact, we believe that, in the context of convexity and numerical implementation in shape optimization, the use of support functions is more advantageous than that of vectors fields. For more details, we refer to [2] where we also gave an example of application. Note also that, in the above statement, we used two deformations of $\Omega_{0}$. The first one, $(1-\varepsilon) \Omega_{0}+\varepsilon \Omega$, was the one considered by A. Niftiyev and Y. Gasimov and was suggested by the convexity context and the applications considered in [1]. This is why we studied it first. Afterwards, we discovered that a formula similar to (2) was known in the classical Brunn-Minkovski theory of convex bodies since a long time for $f=1$ and when one uses the usual deformation $\Omega_{0}+\varepsilon \Omega$ of $\Omega_{0}$. See [9], [6] or [11], for example. This of course gave us a further motivation to study such formulas.

In this paper, we consider again these formulas and extend them yet by removing the assumption on the regularity of $\Omega_{0}$, that is, we establish the above formulas for all domains in $\mathcal{O}$. This is the main result of this work. Besides, as a byproduct of the proof, we obtain other formulas that express the limits in question. We believe that they may be of interest in numerical applications at least because they use an integration domain which is independent of $\Omega_{0}$ and $\Omega$ (see Section 3). Now, let us give an idea on our method of proof. First of all, we have not been able to extend the proof given in [2]. Even if we take again some arguments from that paper (by the bye, not part (iii) of Lemma 8 which is false, see [3]), the spirit is different. We begin by approximating both domains by smooth ones and then work with the approximating sequences and use polar coordinates to transform the main integral. The advantage in doing so is to let the integral depend on the sets only via their gauge functions and only via a one-dimensional integral. By the way, this proof makes a heavy use of gauge functions (see Section 2). To pass to the limit, the main issue is to obtain some compactness. This is solved by means of some fine analysis of the gauge functions : Radon measures, interpolation and BV functions. The result of all that is a limit which is expressed as an integral on $S^{n-1}$ and the last step consists in performing a change of variables to obtain an integral on $\partial \Omega_{0}$.

The outline of the paper is as follows. In Section 2, we recall some facts about convex sets. In Section 3, we state the main result of the paper and also some corollaries. Section 
4 is devoted to the proof of the main result. The last section is an appendix where an interpolation lemma is proved.

Thanks are due to Professor C. Kiselman for confirming us a result about the regularity of the Minkowski sum of convex bodies.

\section{Preliminaries}

Let us recall briefly some notions and facts about convex sets that we shall use in the sequel. For more details or proofs, we refer for example to [9], [4], [6], or [8].

We shall denote by $\mathcal{C}$ the set of closed convex bounded subsets of $\mathbb{R}^{n}$.

Let us start by recalling the operations of addition and multiplication by non-negative numbers in $\mathcal{C}$. If $A$ and $B$ are in $\mathcal{C}$ and $\lambda \geq 0$, then,

$$
\begin{gathered}
A+B=\{x+y ; x \in A, y \in B\} \\
\text { and } \lambda A=\{\lambda x ; \quad x \in A\}
\end{gathered}
$$

are also in $\mathcal{C}$. However, $\mathcal{C}$ is not a linear space.

Instead of it, $\mathcal{C}$ is a metric space if it is equipped with what is called the Hausdorff distance $d^{H}$. This is defined by

$$
d^{H}(A, B)=\inf \{\delta ; \delta>0, A \subset B+B(0, \delta) \text { and } B \subset A+B(0, \delta)\},
$$

where the notation $B(x, r)$ is for the open ball with center $x$ and radius $r$. One can indeed show that $d^{H}$ is a distance on $\mathcal{C}$. One can even show that, provided with the Hausdorff distance, $\mathcal{C}$ is a complete metric space which is also locally compact.

If $A \in \mathcal{C}$, we shall use its support function $P_{A}$ defined in $\mathbb{R}^{n}$ by

$$
P_{A}(x)=\sup _{y \in A} x . y,
$$

where $x . y=x y$ denotes the standard scalar product in $\mathbb{R}^{n}$. One can easily see that the support function $P_{A}$ is continuous, convex and positively homogeneous of degree 1, that is, $P_{A}(\lambda x)=\lambda P_{A}(x), \lambda>0$. Conversely, for each continuous convex positively homogeneous function $P$ on $\mathbb{R}^{n}$, there exists a unique closed convex bounded set $A$, such that $P=P_{A}$. In fact, the set $A \in \mathcal{C}$ is reconstructed as a subdifferential of the function $P$ at the origin $x=0$, that is,

$$
A=\partial P(0)=\left\{y \in \mathbb{R}^{n}: P(x) \geq x . y, \forall x \in \mathbb{R}^{n}\right\} .
$$

Thus, we have a one-one correspondance between $\mathcal{C}$ and the set of all continuous convex positively homogeneous functions $P$ on $\mathbb{R}^{n}$.

The support functions have other remarkable properties. For all $A, B \in \mathcal{C}$ and $\lambda \geq 0$, one can easily prove that

$$
P_{A+B}=P_{A}+P_{B} \quad \text { and } \quad P_{\lambda A}=\lambda P_{A}
$$

An important formula that we shall need and which relates elements of $\mathcal{C}$ with their support functions via the Hausdorff distance is the following :

$$
\sup _{S^{n-1}}\left|P_{A}-P_{B}\right|=d^{H}(A, B), \quad A, B \in \mathcal{C} .
$$


We shall also use what is called the gauge or distance function. This is another function which is associated to a convex set and characterizes it. Let $U$ be an open convex bounded subset of $\mathbb{R}^{n}$ that contains 0 . Then, its gauge function is defined by

$$
F_{U}(x)=\inf \{t>0 ; x \in t U\} .
$$

The following proposition summarizes the main properties of a gauge function.

Proposition 1 If $U$ is an open convex bounded neighbourhood of 0 , then, $F_{U}$ is a non negative continuous convex positively homogeneous function of degree 1. More precisely, we have the following properties :

(i) $F_{U}(0)=0, \quad F_{U}(x)>0, \forall x \neq 0$

(ii) $F_{U}(\lambda x)=\lambda F_{U}(x), \quad \forall \lambda>0, \quad \forall x \in \mathbb{R}^{n}$.

(iii) $F_{U}(x+y) \leq F_{U}(x)+F_{U}(y), \quad \forall x, y \in \mathbb{R}^{n}$.

(iv) $U=\left\{x \in \mathbb{R}^{n} ; F_{U}(x)<1\right\}$ and $\partial U=\left\{x \in \mathbb{R}^{n} ; F_{U}(x)=1\right\}$.

Conversely, any function $F$ which satisfies $(i)$, (ii) and (iii), is the gauge function of the open convex set defined by $U=\{F<1\}$.

Thus, when $U$ is symmetric with respect to $0, F_{U}$ is just the norm whose open unit ball is $U$. For a proof of this proposition, we refer for example to [6].

One can also show that the regularity of $U$ is equivalent to that of $F_{U}$, that is, $U$ is a $C^{k}$ domain if and only if $F_{U}$ is of class $C^{k}$ in $\mathbb{R}^{n} \backslash\{0\}$. A proof of this fact is given in [2]. is

The analogue of (5) for gauge functions is merely an inequality and we shall need it. This

Proposition 2 If $A$ and $B$ are convex, compact and contain the ball $\bar{B}(0, r), r>0$, we have the inequality

$$
\sup _{S^{n-1}}\left|F_{A}-F_{B}\right| \leq \frac{1}{r^{2}} d^{H}(A, B)
$$

We shall also crucially need the following result which essentially says that one can estimate derivatives of a gauge function by the function itself.

Proposition 3 Let $U$ be an open convex bounded neighbourhood of 0 and $F_{U}$ its gauge function. Then,

(i) $\nabla F_{U}$ exists almost everywhere in $\mathbb{R}^{n}$ and in the sense of distributions and is in $L^{\infty}\left(\mathbb{R}^{n}\right)$. More precisely, we have almost everywhere

$$
\left|\nabla F_{U}(x)\right| \leq \sqrt{n} \sup _{S^{n-1}} F_{U}
$$

(ii) The second partial distributional derivatives of $F_{U}$ are Radon measures that we can estimate for any bounded open subset $D$ of $\mathbb{R}^{n}$ as follows :

$$
\left|\left\langle\partial_{i} \partial_{j} F_{U}, \varphi\right\rangle\right| \leq C_{D} \sup _{D^{\prime}} F_{U}\|\varphi\|_{L^{\infty}}, \quad 1 \leq i, j \leq n
$$

for all smooth functions $\phi$ with compact support in $D$ (notation : $\varphi \in \mathscr{D}(D)$ ), where the constant $C_{D}$ depends only on the set $D$ and $D^{\prime}$ is a domain which is slightly larger than $D$. 
Proof : $(i)$ By Rademacher's theorem, it suffices to show that $F_{U}$ is a Lipschitz function and to estimate the Lipschitz constant. By Proposition 1, we have

$$
\left.F_{U}(x)=F_{U}(x-y+y)\right] \leq F_{U}(x-y)+F_{U}(y) \leq|x-y| \sup _{S^{n-1}} F_{U}+F_{U}(y),
$$

for all $x, y \in \mathbb{R}^{n}$. This yields :

$$
\left|F_{U}(x)-F_{U}(y)\right| \leq C|x-y|, \quad x, y \in \mathbb{R}^{n},
$$

with $C=\sup _{S^{n-1}} F_{U}$, which implies the result.

(ii) It follows from the convexity of the function $F_{U}$ that its distributional hessian $F_{U}^{\prime \prime}$ is a non negative Radon measure in $\mathbb{R}^{n}$, that is, for all $h \in \mathbb{R}^{n}$ and all non negative $\varphi \in \mathscr{D}\left(\mathbb{R}^{n}\right)$, we have

$$
\left\langle{ }^{t} h F_{U}^{\prime \prime} h, \varphi\right\rangle \geq 0
$$

This extends the usual property that says that the hessian of a convex function of class $C^{2}$ in a non negative matrix. See [7], Theorem 2, page 239. Moreover, the norm on a compact set of such a non negative Radon measure can be estimated simply by the value of the measure on a test function $\chi$ which is 1 on the compact set, that is,

$$
\left|\left\langle{ }^{t} h F_{U}^{\prime \prime} h, \varphi\right\rangle\right| \leq\left\langle{ }^{t} h F_{U}^{\prime \prime} h, \chi\right\rangle\|\varphi\|_{L^{\infty}}
$$

for all $\varphi \in \mathscr{D}\left(\mathbb{R}^{n}\right), \operatorname{supp}(\varphi) \subset K, K$ is a compact set in $\mathbb{R}^{n}$ and $\chi \in \mathscr{D}\left(\mathbb{R}^{n}\right), \chi \geq 0, \chi=1$ on $K$. See [7], Corollary 1 , page 53. Denoting by $e_{i}, 1 \leq i \leq n$, the canonical basis vectors of $\mathbb{R}^{n}$, we can write $\partial_{i} \partial_{j} F_{U}=\left({ }^{t}\left(e_{i}+e_{j}\right) F_{U}^{\prime \prime}\left(e_{i}+e_{j}\right)-{ }^{t} e_{i} F_{U}^{\prime \prime} e_{i}-{ }^{t} e_{j} F_{U}^{\prime \prime} e_{j}\right) / 2$. Then, it follows from (7) applied with $K=\bar{D}$ that

$$
\left|\left\langle\partial_{i} \partial_{j} F_{U}, \varphi\right\rangle\right| \leq\left\langle\partial_{i}^{2} F_{U}+\partial_{i} \partial_{j} F_{U}+\partial_{j}^{2} F_{U}, \chi\right\rangle\|\varphi\|_{L^{\infty}}=\left\langle F_{U}, \partial_{i}^{2} \chi+\partial_{i} \partial_{j} \chi+\partial_{j}^{2} \chi\right\rangle\|\varphi\|_{L^{\infty}},
$$

for all $\varphi \in \mathscr{D}(D)$. Hence,

$$
\left|\left\langle\partial_{i} \partial_{j} F_{U}, \varphi\right\rangle\right| \leq C_{D} \sup _{D^{\prime}} F_{U}\|\varphi\|_{L^{\infty}}
$$

with $C_{D}=\left\|\partial_{i}^{2} \chi+\partial_{i} \partial_{j} \chi+\partial_{j}^{2} \chi\right\|_{L^{1}}$

\section{Statement of the results}

Recall that $\mathcal{O}$ is the set of non empty bounded convex open subsets of $\mathbb{R}^{n}$. If $\Omega \in \mathcal{O}$, its closure $\bar{\Omega}$ is in $\mathcal{C}$ and its support function $P_{\bar{\Omega}}$ is well-defined. Since $\sup _{y \in \Omega} x y=\sup _{y \in \bar{\Omega}} x y$ and in order to simplify, we shall use the notation $P_{\Omega}$ instead of $P_{\bar{\Omega}}$. We can now state the main result of this paper. It concerns the derivative of the shape functional

$$
J(\Omega)=\int_{\Omega} f d x
$$

and reads as follows. 
Theorem 1 For all $f \in W_{\text {loc }}^{1,1}\left(\mathbb{R}^{n}\right)$ and $\Omega_{0}, \Omega \in \mathcal{O}$, we have the limit

$$
\lim _{t \rightarrow 0^{+}} \frac{J\left(\Omega_{0}+t \Omega\right)-J\left(\Omega_{0}\right)}{t}=\int_{\partial \Omega_{0}} f(x) P_{\Omega}\left(\nu_{0}(x)\right) d \sigma(x),
$$

and, if $\Omega_{0}$ is a neighbourhood of 0 , we also have

$$
\lim _{t \rightarrow 0^{+}} \frac{J\left(\Omega_{0}+t \Omega\right)-J\left(\Omega_{0}\right)}{t}=\int_{S^{n-1}} f\left(\frac{\omega}{F_{\Omega_{0}}(\omega)}\right) P_{\Omega}\left(\nabla F_{\Omega_{0}}(\omega)\right) \frac{d \omega}{F_{\Omega_{0}}(\omega)^{n}},
$$

where $\nu_{0}(x)$ denotes the exterior unit normal vector to $\partial \Omega_{0}$ at $x, P_{\Omega}$ is the support function of $\Omega$ and $F_{\Omega_{0}}$ is the gauge function of $\Omega_{0}$.

Recall that, as a convex domain, $\Omega_{0}$ is a Lipschitz domain and this implies that $\nu_{0}(x)$ exists on $\partial \Omega_{0}$ almost everywhere with respect to the usual boundary measure.

Note also that in the above statement we consider only the limit with respect to the standard deformation $\Omega_{0}+t \Omega$, the limit (2) being in fact considered in Corollary 2 below. Instead, in Theorem 1, we have given two expressions for the limit. What happened is that the method of proof gives first the second one (10) and the first one is then obtained by a change of variables. Our opinion is that this (more or less) new expression may be useful in numerical analysis, even if we have not yet tested it, because of the fixed domain of integration and because of the dependance on the domains which occurs only via the support and gauge functions.

The proof of Theorem 1 will be given in next section. Before, let us state and prove two corollaries. The first one may also be regarded as an extension of it. This is the case where the function $f$ itself depends on the parameter $t$, a situation which is frequent in the applications.

Corollary 1 Let $\Omega_{0}, \Omega \in \mathcal{O}$ and let $\Omega_{t}$ stands for $\Omega_{0}+t \Omega, 0 \leq t \leq 1$. Let $f_{t}, 0 \leq t \leq 1$, be a family of functions in $L_{l o c}^{1}\left(\mathbb{R}^{n}\right)$ such that $f_{0} \in W_{l o c}^{1,1}\left(\mathbb{R}^{n}\right)$ and assume that the limit

$$
g=\lim _{t \rightarrow 0^{+}} \frac{f_{t}-f_{0}}{t}
$$

exists in $L_{\text {loc }}^{1}\left(\mathbb{R}^{n}\right)$ or, at least, in $L^{1}(D)$, where $D$ is a bounded open set in $\mathbb{R}^{n}$ which contains all the $\Omega_{t}$. Then, the (right) derivative at 0 of $I(t)=\int_{\Omega_{t}} f_{t} d x$ exists and is equal to

$$
I^{\prime}(0)=\int_{\Omega_{0}} g(x) d x+\int_{\partial \Omega_{0}} f_{0}(x) P_{\Omega}\left(\nu_{0}(x)\right) d \sigma(x),
$$

and, if $\Omega_{0}$ is a neighbourhood of 0 , it is also equal to

$$
\int_{\Omega_{0}} g(x) d x+\int_{S^{n-1}} f_{0}\left(\frac{\omega}{F_{\Omega_{0}}(\omega)}\right) P_{\Omega}\left(\nabla F_{\Omega_{0}}(\omega)\right) \frac{d \omega}{F_{\Omega_{0}}(\omega)^{n}} .
$$

Proof of Corollary 1 : The proof follows the same arguments as that of Corollary 1 of [2], so we refer to it. Of course, to get (11), we have just to use the expression (10) instead of (9) in the proof.

The second corollary concerns the case where one uses the deformation considered by [1], that is, $(1-t) \Omega_{0}+t \Omega$, and treats at the same time the case where the function $f$ depends on the parameter $t$. 
Corollary 2 Let $\Omega_{0}, \Omega \in \mathcal{O}$ and let $\Omega_{t}$ stands for $(1-t) \Omega_{0}+t \Omega, 0 \leq t \leq 1$. Let $f_{t}, 0 \leq t \leq 1$, be a family of functions in $L_{\text {loc }}^{1}\left(\mathbb{R}^{n}\right)$ such that $f_{0} \in W_{\text {loc }}^{1,1}\left(\mathbb{R}^{n}\right)$ and assume that the limit

$$
g=\lim _{t \rightarrow 0^{+}} \frac{f_{t}-f_{0}}{t}
$$

exists in $L_{\text {loc }}^{1}\left(\mathbb{R}^{n}\right)$ or, at least, in $L^{1}(D)$, where $D$ is a bounded open set in $\mathbb{R}^{n}$ which contains all the $\Omega_{t}$. Then, the (right) derivative at 0 of $I(t)=\int_{\Omega_{t}} f_{t} d x$ exists and is equal to

$$
I^{\prime}(0)=\int_{\Omega_{0}} g(x) d x+\int_{\partial \Omega_{0}} f_{0}(x)\left(P_{\Omega}\left(\nu_{0}(x)\right)-P_{\Omega_{0}}\left(\nu_{0}(x)\right)\right) d \sigma(x),
$$

and, if $\Omega_{0}$ is a neighbourhood of 0 , it is also equal to

$$
\int_{\Omega_{0}} g(x) d x+\int_{S^{n-1}} f_{0}\left(\frac{\omega}{F_{\Omega_{0}}(\omega)}\right)\left[P_{\Omega}\left(\nabla F_{\Omega_{0}}(\omega)\right)-P_{\Omega_{0}}\left(\nabla F_{\Omega_{0}}(\omega)\right)\right] \frac{d \omega}{F_{\Omega_{0}}(\omega)^{n}} .
$$

Proof of Corollary 2 : By the same argument as that used to prove Corollary 1, it is sufficient to consider the case where $f_{t}=f_{0}$ does not depend on $t$.

We can write $\Omega_{t}=(1-t)\left(\Omega_{0}+s \Omega\right)$ with $s=t /(1-t)$, so that, by an obvious change of variables,

$$
\frac{J\left(\Omega_{t}\right)-J\left(\Omega_{0}\right)}{t}=\frac{1+s}{s}\left(\int_{\Omega_{0}+s \Omega} f_{s} d x-\int_{\Omega_{0}} f_{0} d x\right) \text { where } f_{s}(x)=f_{0}\left(\frac{x}{1+s}\right)\left(\frac{1}{1+s}\right)^{n} .
$$

Now, as $s \rightarrow 0$, we have

$$
\frac{f_{s}-f_{0}}{s} \longrightarrow-n f_{0}(x)-f_{0}^{\prime}(x) x=-\operatorname{div}\left(f_{0}(x) x\right) \quad \text { in } \quad L_{l o c}^{1}
$$

Indeed, this follows from the application of the following more or less known lemma :

Lemma 1 Let $\left(\Phi_{t}\right)_{|t| \leq \epsilon_{0}}$ be a family of $C^{1}$ diffeomorphisms from $\mathbb{R}^{n}$ onto $\mathbb{R}^{n}$ such that $\Phi_{0}(x)=x$ and $(t, x) \mapsto \Phi_{t}(x)$ and $(t, y) \mapsto \Phi_{t}^{-1}(y)$ are of class $C^{1}$ in $\left[-\epsilon_{0}, \epsilon_{0}\right] \times \mathbb{R}^{n}$. Then, for all $f \in W_{\text {loc }}^{1,1}\left(\mathbb{R}^{n}\right)$, the limit $\lim _{t \rightarrow 0}\left(f\left(\Phi_{t}(x)\right)-f(x)\right) / t$ exists in $L_{l o c}^{1}\left(\mathbb{R}^{n}\right)$ and is equal to $\left.f^{\prime}(x) \cdot \frac{d}{d t} \Phi_{t}(x)\right|_{t=0}$.

For a proof of this lemma, see [5], Chapter 5. Then, applying Corollary 1 yields the formula

$$
\begin{aligned}
\lim _{t \rightarrow 0^{+}} \frac{J\left(\Omega_{t}\right)-J\left(\Omega_{0}\right)}{t} & =\int_{\partial \Omega_{0}} f_{0}(x) P_{\Omega}\left(\nu_{0}(x)\right) d \sigma(x)-\int_{\Omega_{0}} \operatorname{div}\left(f_{0}(x) x\right) d x \\
& =\int_{\partial \Omega_{0}} f_{0}(x)\left(P_{\Omega}\left(\nu_{0}(x)\right)-P_{\Omega_{0}}\left(\nu_{0}(x)\right) d \sigma(x)\right.
\end{aligned}
$$

where the last equality is obtained by applying the divergence formula and by noting that $x \nu_{0}(x)=P_{\Omega_{0}}\left(\nu_{0}(x)\right)$. To get (12), one can for example apply the change of variables used at the end of proof of Theorem 1 which transforms an integral on $\partial \Omega_{0}$ into an integral on $S^{n-1}$ (see section 4). 


\section{Proof of Theorem 1}

Let $f \in W_{\text {loc }}^{1,1}\left(\mathbb{R}^{n}\right), \Omega_{0}, \Omega \in \mathcal{O}$ and let $\Omega_{t}$ stands for $\Omega_{0}+t \Omega$ where $t \in[0,1]$. The proof relies on an analysis using heavily the gauge functions of $\Omega_{0}, \Omega$ and $\Omega_{t}$ which we shall denote by $F_{0}, F$ and $F_{t}$ respectively. This assumes that $\Omega_{0}$ and $\Omega$ are neighborhoods of 0 , but this is not a restriction of generality. Indeed, assume that Theorem 1 is proved in this case, then, if $a, b \in \mathbb{R}^{n}$, we have, by obvious changes of variables,

$$
\begin{aligned}
\left(J\left(a+\Omega_{0}+t(b+\Omega)\right)-J(a\right. & \left.\left.+\Omega_{0}\right)\right) / t=\left(J\left(a+t b+\Omega_{t}\right)-J\left(a+\Omega_{0}\right)\right) / t \\
= & \frac{1}{t}\left(\int_{\Omega_{t}} f(a+t b+x) d x-\int_{\Omega_{0}} f(a+x) d x\right) .
\end{aligned}
$$

It follows then from Lemma 1 that

$$
\frac{f(x+a+t b)-f(x+a)}{t} \longrightarrow f^{\prime}(x+a) \cdot b=\operatorname{div}(f(x+a) b) \quad \text { in } \quad L_{l o c}^{1}\left(\mathbb{R}^{n}\right)
$$

as $t \rightarrow 0^{+}$, and from Corollary 1 that

$$
\lim _{t \rightarrow 0^{+}} \frac{J\left(a+\Omega_{0}+t(b+\Omega)-J\left(a+\Omega_{0}\right)\right.}{t}=\int_{\partial \Omega_{0}} f(x+a) P_{\Omega}\left(\nu_{0}(x)\right) d \sigma(x)+\int_{\Omega_{0}} \operatorname{div}(f(x+a) b) d x .
$$

Now, it remains to apply the divergence formula to get

$$
\begin{aligned}
\lim _{t \rightarrow 0^{+}} \frac{J\left(a+\Omega_{0}+t(b+\Omega)-J\left(a+\Omega_{0}\right)\right.}{t} & =\int_{\partial \Omega_{0}} f(x+a) P_{\Omega}\left(\nu_{0}(x)\right) d \sigma+\int_{\partial \Omega_{0}} f(x+a) b . \nu_{0}(x) d \sigma \\
& =\int_{\partial \Omega_{0}} f(x+a) P_{b+\Omega}\left(\nu_{0}(x)\right) d \sigma \\
& =\int_{\partial\left(a+\Omega_{0}\right)} f(x) P_{b+\Omega}\left(\nu_{a+\Omega_{0}}(x)\right) d \sigma
\end{aligned}
$$

where $\nu_{a+\Omega_{0}}$ is the exterior unit normal vector to $\partial\left(a+\Omega_{0}\right)$ at $x$, which establishes the formula in the case where the domains are not necessarily neighbourhoods of 0 .

The first idea in the actual proof of Theorem 1 is to compute the derivative of $t \mapsto F_{t}$. We shall see later on how this can be exploited. The proof uses part of the construction made in [2] and we refer to it for more details.

Lemma 2 Assume that $\Omega_{0}$ and $\Omega$ are smooth and that $\Omega$ is strongly convex. Then, the function $(t, x) \mapsto F_{t}(x)$ is smooth at least in $[0,1] \times\left(\mathbb{R}^{n} \backslash 0\right)$ and we have

$$
\frac{d}{d t} F_{t}(x)=-F_{t}(x) P_{\Omega}\left(\nabla F_{t}(x)\right)
$$

where $P_{\Omega}$ is the support function of $\Omega$.

Proof : Under the assumptions of the lemma, it follows from the construction done in [2] that, at least for small $t$, there exists a Lipschitz homeomorphism $\Phi_{t}: \mathbb{R}^{n} \rightarrow \mathbb{R}^{n}$ which is smooth away from 0 and such that $\Phi_{t}\left(\Omega_{0}\right)=\Omega_{t}$. Moreover, $\Phi_{t}$ has the form $\Phi_{t}(x)=x+t a(x)$ where $a: \mathbb{R}^{n} \rightarrow \mathbb{R}^{n}$ is homogeneous of degree 1 . We remark here that even if the deformation $\Omega_{t}=\Omega_{0}+t \Omega$ is not exactly that used in [2], that is, $\Omega_{t}=(1-t) \Omega_{0}+t \Omega$, the construction 
works as well with obvious modifications. It is then easy to see that $F_{t}(x)=F_{0}\left(\Phi_{t}^{-1}(x)\right)$. Since $x \mapsto \Phi_{t}(x)$ is a proper map (at least for small $t$ ), then $(t, x) \mapsto \Phi_{t}^{-1}(x)$ is continuous and it is also smooth for small $t$ and $x \neq 0$. Hence, $(t, x) \mapsto F_{t}(x)$ is also smooth and we can compute the wanted derivative by using the identity $F_{0}(x)=F_{t}\left(\Phi_{t}(x)\right)$. Indeed, taking the derivative with respect to $t$ of both members of this equality, we get

$$
0=\left(\frac{d}{d t} F_{t}\right)\left(\Phi_{t}(x)\right)+\nabla F_{t}\left(\Phi_{t}(x)\right) \frac{d}{d t} \Phi_{t}(x)=\left(\frac{d}{d t} F_{t}\right)\left(\Phi_{t}(x)\right)+\nabla F_{t}\left(\Phi_{t}(x)\right) a(x) .
$$

Hence,

$$
\frac{d}{d t} F_{t}(x)=-\nabla F_{t}(x) a\left(\Phi_{t}^{-1}(x)\right) .
$$

In particular, $\left.\frac{d}{d t} F_{t}(x)\right|_{t=0}=-\nabla F_{0}(x) a(x)$. Now, it follows from Lemma 1 of [2] that $P_{\Omega}\left(\nu_{0}(x)\right)=\nu_{0}(x) a(x)$ for all $x \in \partial \Omega_{0}$, where $\nu_{0}$ is the outward unit normal vector field to $\Omega_{0}$. Since $\nu_{0}(x)=\nabla F_{0}(x) /\left|\nabla F_{0}(x)\right|$, this implies

$$
\left.\frac{d}{d t} F_{t}(x)\right|_{t=0}=-\left|\nabla F_{0}(x)\right| P_{\Omega}\left(\nu_{0}(x)\right)=-P_{\Omega}\left(\nabla F_{0}(x)\right), \quad x \in \partial \Omega_{0} .
$$

Now, if $x \neq 0$, then $x / F_{0}(x)$ is on $\partial \Omega_{0}$, and since $x \mapsto \frac{d}{d t} F_{t}(x)$ is homogeneous of degree 1 , we obtain

$$
\left.\frac{d}{d t} F_{t}(x)\right|_{t=0}=-F_{0}(x) P_{\Omega}\left(\nabla F_{0}(x)\right)
$$

To get the formula for $t>0$, note that $\Omega_{t+h}=\Omega_{t}+h \Omega$ by convexity of $\Omega$ (with $h>0$ ), and that $\Omega_{t}$ is also smooth since $\Omega$ is strongly convex. Therefore, since $F_{t+h}$ is obtained from $F_{t}$ like $F_{t}$ is obtained from $F_{0}$, we get

$$
\frac{d}{d t} F_{t}(x)=\left.\frac{d}{d h} F_{t+h}(x)\right|_{h=0}=-F_{t}(x) P_{\Omega}\left(\nabla F_{t}(x)\right) .
$$

The next step is to remove the smoothness assumptions made on $\Omega_{0}$ and $\Omega$ in the above lemma and it will be the longest and most important step in the proof of Theorem 1 . This is done by approximation.

Lemma 3 If $\Omega_{0}, \Omega \in \mathcal{O}$ and are neighborhoods of 0 , then, for all $t \in[0,1]$ and almost every $x \in S^{n-1}$, we have

$$
F_{t}(x)-F_{0}(x)=-\int_{0}^{t} F_{s}(x) P_{\Omega}\left(\nabla F_{s}(x)\right) d s .
$$

Proof : It is a classical fact that one can approximate a convex body by smooth and strongly convex ones in the sense of the Hausdorff distance $d^{H}$. See for example [9] or [6]. So, let $\left(\Omega_{0}^{k}\right)$ and $\left(\Omega^{k}\right)$ be sequences in $\mathcal{O}$ such that, for all $k, \Omega_{0}^{k}$ and $\Omega^{k}$ are of class $C^{2}$, and $\Omega^{k}$ is strongly convex, and such that $d^{H}\left(\overline{\Omega_{0}^{k}}, \overline{\Omega_{0}}\right) \rightarrow 0$ and $d^{H}\left(\overline{\Omega^{k}}, \bar{\Omega}\right) \rightarrow 0$ as $k \rightarrow \infty$. Let $\Omega_{t}^{k}$ stands for $\Omega_{0}^{k}+t \Omega^{k}$ and let $F_{0}^{k}, F^{k}$ and $F_{t}^{k}$ denote the gauge functions of $\Omega_{0}^{k}, \Omega^{k}$ and $\Omega_{t}^{k}$ respectively, $t \in[0,1]$. It follows from Lemma 1 that, for all $k \in \mathbb{N}, t \in[0,1]$ and $x \in \mathbb{R}^{n}$, we have

$$
\frac{d}{d t} F_{t}^{k}(x)=-F_{t}^{k}(x) P_{\Omega^{k}}\left(\nabla F_{t}^{k}(x)\right)
$$


so that

$$
F_{t}^{k}(x)-F_{0}^{k}(x)=-\int_{0}^{t} F_{s}^{k}(x) P_{\Omega^{k}}\left(\nabla F_{s}^{k}(x)\right) d s .
$$

Of course, we wish to pass to the limit when $k \rightarrow \infty$ in this expression.

Let us first deal with the left hand side of (14). Note that it is easy to take all the sets $\Omega_{0}, \Omega, \Omega_{0}^{k}, \Omega^{k}, \Omega_{t}$ and $\Omega_{t}^{k}$ such that they contain the same (may be small) fixed ball $\bar{B}(0, r)$, $r>0$. Hence, it follows from (6), (5) and (4) that

$$
\sup _{S^{n-1}}\left|F_{t}^{k}-F_{t}\right| \leq \frac{1}{r^{2}} d^{H}\left(\overline{\Omega_{t}^{k}}, \overline{\Omega_{t}}\right)=\frac{1}{r^{2}} \sup _{S^{n-1}}\left|P_{\Omega_{t}^{k}}-P_{\Omega_{t}}\right| \leq \frac{1+t}{r^{2}}\left(d^{H}\left(\overline{\Omega_{0}^{k}}, \overline{\Omega_{0}}\right)+d^{H}\left(\overline{\Omega^{k}}, \bar{\Omega}\right)\right) .
$$

This means that, when $k \rightarrow \infty, F_{t}^{k}(x)$ converges to $F_{t}(x)$ uniformly when $(t, x)$ is in a compact set. Thus, $F_{t}^{k}(x)-F_{0}^{k}(x)$ converges uniformly on compact sets to $F_{t}(x)-F_{0}(x)$.

The right hand side of (14) needs a finer and more elaborate analysis. We are concerned with the convergence of the sequence of gradients $\left(\nabla F_{t}^{k}\right)$ and the problem is that, a priori, what is available is just some weak convergence and, in particular, the convergence in the sense of distributions, a convergence which we can not compose with $P_{\Omega^{k}}$. However, by exploiting the full strength of the convexity, we are going to highlight a quite satisfying property of convergence for that sequence which will allow us to conclude.

In fact, let us show that the sequence $\left(\nabla F_{t}^{k}\right)$ is relatively compact in the space $E=$ $C\left([0,1], L^{1}(D)\right)$, where $D$ is a large bounded domain which contains all the sets under study : $\Omega_{0}, \Omega, \Omega_{0}^{k}, \Omega^{k}, \Omega_{t}$ and $\Omega_{t}^{k}$; for example, $D=B(0, R)$ with a large enough $R$. This will be done by means of Ascoli-Arzela's theorem. To begin, let us establish some uniform estimates. First, applying (6), (5) and (4), we have the estimate

$$
\left|F_{t_{1}}^{k}(x)-F_{t_{2}}^{k}(x)\right| \leq \frac{|x|}{r^{2}} d^{H}\left(\overline{\Omega_{t_{1}}^{k}}, \overline{\Omega_{t_{2}}^{k}}\right)=\frac{|x|}{r^{2}} \sup _{S^{n-1}}\left|P_{\Omega_{t_{1}}^{k}}-P_{\Omega_{t_{2}}^{k}}\right|=\frac{|x|}{r^{2}}\left|t_{1}-t_{2}\right| \sup _{S^{n-1}} P_{\Omega^{k}} .
$$

Hence,

$$
\left|F_{t_{1}}^{k}(x)-F_{t_{2}}^{k}(x)\right| \leq \frac{|x|}{r^{2}}\left|t_{1}-t_{2}\right| \sup _{S^{n-1}} P_{D}, \quad x \in \mathbb{R}^{n}, t_{1}, t_{2} \in[0,1] .
$$

On the other hand, it follows from part $(i i)$ of Proposition 2 that, for $1 \leq i, j \leq n$,

$$
\left|\left\langle\partial_{i} \partial_{j}\left(F_{t_{1}}^{k}-F_{t_{2}}^{k}\right), \varphi\right\rangle\right| \leq C_{D} \sup _{D^{\prime}}\left(F_{t_{1}}^{k}+F_{t_{2}}^{k}\right)\|\varphi\|_{L^{\infty}} \leq 2 C_{D} \sup _{D^{\prime}} F_{B(0, r)}\|\varphi\|_{L^{\infty}},
$$

where $D^{\prime}$ is slightly larger than $D$, so that,

$$
\left|\left\langle\partial_{i} \partial_{j}\left(F_{t_{1}}^{k}-F_{t_{2}}^{k}\right), \varphi\right\rangle\right| \leq C_{D, r}^{\prime}\|\varphi\|_{L^{\infty}}, \quad \varphi \in \mathscr{O}(D), t_{1}, t_{2} \in[0,1] .
$$

We need now the following interpolation lemma whose proof is postponed to the appendix.

Lemma 4 Let $T$ be a tempered distribution in $\mathbb{R}^{n}$ which satisfies the estimates

$$
\begin{gathered}
|\langle T, \varphi\rangle| \leq C_{1}\|\varphi\|_{L^{\infty}}, \\
\left|\left\langle\partial_{i} \partial_{j} T, \varphi\right\rangle\right| \leq C_{2}\|\varphi\|_{L^{\infty}},
\end{gathered}
$$

for all $\varphi \in \mathscr{D}\left(\mathbb{R}^{n}\right), 1 \leq i, j \leq n$. Then, there exists a constant $C$ which depends only on the dimension $n$ such that

$$
\left|\left\langle\partial_{j} T, \varphi\right\rangle\right| \leq C \sqrt{C_{1} C_{2}}\|\varphi\|_{L^{\infty}}, \quad \varphi \in \mathscr{Q}\left(\mathbb{R}^{n}\right), 1 \leq j \leq n .
$$


Obviously, (17) implies

$$
\left|\left\langle F_{t_{1}}^{k}-F_{t_{2}}^{k}, \varphi\right\rangle\right| \leq C_{D, r}^{\prime \prime}\left|t_{1}-t_{2}\right|\|\varphi\|_{L^{\infty}}, \quad \varphi \in \mathscr{O}(D), t_{1}, t_{2} \in[0,1],
$$

and, of course, we would like to apply the above lemma to the tempered distribution $F_{t_{1}}^{k}-F_{t_{2}}^{k}$. In fact, we apply it to $T=\psi\left(F_{t_{1}}^{k}-F_{t_{2}}^{k}\right)$, where $\psi \in \mathscr{O}\left(D^{\prime}\right)$ is such that $\psi=1$ on $\bar{D}$ and $D^{\prime}$ is a domain slightly larger than $D$ and, clearly, (19) and (18) also hold in $D^{\prime}$. The result is that we have, for all $\varphi \in \mathscr{D}(D), t_{1}, t_{2} \in[0,1], 1 \leq i \leq n$,

$$
\left|\left\langle\partial_{i}\left(F_{t_{1}}^{k}-F_{t_{2}}^{k}\right), \varphi\right\rangle\right| \leq C\left(C_{D, r}^{\prime} C_{D, r}^{\prime \prime}\left|t_{1}-t_{2}\right|\right)^{1 / 2}\|\varphi\|_{L^{\infty}}
$$

from which we can deduce that

$$
\int_{D}\left|\partial_{i}\left(F_{t_{1}}^{k}-F_{t_{2}}^{k}\right)\right| d x \leq C^{\prime}\left|t_{1}-t_{2}\right|^{1 / 2}, \quad t_{1}, t_{2} \in[0,1], 1 \leq i \leq n,
$$

where $C^{\prime}$ does not depend on $k$. Of course, (21) is a continuity estimate for $\nabla F_{t}^{k}$. More precisely, it says that $t \mapsto \nabla F_{t}^{k}$ is Hölder continuous, and since the Hölder constant $C^{\prime}$ does not depend on $k$, the sequence $\left(\nabla F_{t}^{k}\right)$ is equicontinuous in $E$.

It remains to show that, for all $t \in[0,1]$, the sequence $\left(\nabla F_{t}^{k}\right)$ is relatively compact in $L^{1}(D)$. It follows from Proposition 2 that

$$
\left\|\nabla F_{t}^{k}\right\|_{L^{1}(D)} \leq \operatorname{meas}(D) \sup _{S^{n-1}} F_{t}^{k} \leq \operatorname{meas}(D) \sup _{S^{n-1}} F_{B(0, r)}=\frac{\operatorname{meas}(D)}{r}
$$

and that, for all $\varphi \in \mathscr{O}(D), 1 \leq i, j \leq n$,

$$
\left|\left\langle\partial_{i} \partial_{j} F_{t}^{k}, \varphi\right\rangle\right| \leq C_{D} \sup _{D} F_{t}^{k}\|\varphi\|_{L^{\infty}} \leq C_{D} \sup _{D} F_{B(0, r)}\|\varphi\|_{L^{\infty}}=\frac{C_{D}^{\prime}}{r}\|\varphi\|_{L^{\infty}}
$$

which shows that the sequence $\left(\nabla F_{t}^{k}\right)$ is bounded in the space $B V(D)$ of functions of bounded variation in $D$. It follows then from a well known result (see [7], Theorem 4, page 174) that $\left(\nabla F_{t}^{k}\right)$ is relatively compact in $L^{1}(D)$.

Now, by virtue of Ascoli-Arzela's theorem, the sequence of functions $(t, x) \mapsto \nabla F_{t}^{k}(x)$ is relatively compact in $E$. So, we can find a subsequence which is convergent to some $G_{t}(x)$ in $E$. Since we already know that, for all $t$, the sequence converges in the distributional sense (in $\mathbb{R}^{n}$ ) to $\nabla F_{t}$, we deduce that $G_{t}(x)=\nabla F_{t}(x)$.

Finally, we are able to pass to the limit in the right hand side of (14). Indeed, we already know that the factor $F_{s}^{k}(x)$ converges uniformly, say on $[0,1] \times D$, to $F_{s}(x)$. As for the other factor, we have

$$
\begin{aligned}
\left|P_{\Omega^{k}}\left(\nabla F_{s}^{k}\right)-P_{\Omega}\left(\nabla F_{s}\right)\right| & \leq\left|\left(P_{\Omega^{k}}-P_{\Omega}\right)\left(\nabla F_{s}^{k}\right)\right|+\left|P_{\Omega}\left(\nabla F_{s}^{k}\right)-P_{\Omega}\left(\nabla F_{s}\right)\right| \\
& \leq\left|\nabla F_{s}^{k}\right| d^{H}\left(\overline{\Omega^{k}}, \bar{\Omega}\right)+C\left|\nabla F_{s}^{k}-\nabla F_{s}\right| \\
& \leq \frac{1}{r} d^{H}\left(\overline{\Omega^{k}}, \bar{\Omega}\right)+C\left|\nabla F_{s}^{k}-\nabla F_{s}\right|
\end{aligned}
$$

which implies that, for all $t \in[0,1]$, when $k \rightarrow \infty$,

$$
\int_{0}^{t}\left|P_{\Omega^{k}}\left(\nabla F_{s}^{k}\right)-P_{\Omega}\left(\nabla F_{s}\right)\right| d s \longrightarrow 0
$$


in $L^{1}(D)$ and hence, in $L^{1}\left(S^{n-1}\right)$ by homogeneity. We obtain in fine, for all $t \in[0,1]$ and almost every $x \in S^{n-1}$,

$$
F_{t}(x)-F_{0}(x)=-\int_{0}^{t} F_{s}(x) P_{\Omega}\left(\nabla F_{s}(x)\right) d s .
$$

End of proof of Theorem 1 : We are concerned with the limit when $t \rightarrow 0^{+}$of the expression

$$
I_{t}=\frac{1}{t}\left(\int_{\Omega_{t}} f(x) d x-\int_{\Omega_{0}} f(x) d x\right)
$$

Using polar coordinates, we can write

$$
I_{t}=\frac{1}{t} \int_{S^{n-1}} \int_{0}^{1 / F_{t}(\omega)} f(\varrho \omega) \varrho^{n-1} d \varrho d \omega-\frac{1}{t} \int_{S^{n-1}} \int_{0}^{1 / F_{0}(\omega)} f(\varrho \omega) \varrho^{n-1} d \varrho d \omega=\int_{S^{n-1}} g_{t}(\omega) d \omega
$$

where

$$
g_{t}(\omega)=\frac{1}{t} \int_{1 / F_{0}(\omega)}^{1 / F_{t}(\omega)} f(\varrho \omega) \varrho^{n-1} d \varrho
$$

It follows from the assumption and Fubini's theorem that $\varrho \mapsto f(\varrho \omega) \varrho^{n-1}$ is in $W_{l o c}^{1,1}(\mathbb{R})$ (which is contained in $C(\mathbb{R})$ ) for a.e. $\omega \in S^{n-1}$ and from the fundamental theorem of calculus that

$$
\lim _{h \rightarrow 0} \frac{1}{h} \int_{\sigma}^{\sigma+h} f(\varrho \omega) \varrho^{n-1} d \varrho=f(\sigma \omega) \sigma^{n-1},
$$

for a.e. $\omega \in S^{n-1}$. The idea is to apply such a formula to $g_{t}(\omega)$ which has a similar form. In fact, one can write

$$
\begin{aligned}
g_{t}(\omega) & =\frac{F_{0}(\omega)-F_{t}(\omega)}{t} \frac{1}{F_{0}(\omega) F_{t}(\omega)} \frac{1}{h_{t}(\omega)} \int_{1 / F_{0}(\omega)}^{1 / F_{t}(\omega)} f(\varrho \omega) \varrho^{n-1} d \varrho, \\
& =\frac{1}{F_{0}(\omega) F_{t}(\omega)} \int_{0}^{1} F_{s t}(\omega) P_{\Omega}\left(\nabla F_{s t}(\omega)\right) d s \frac{1}{h_{t}(\omega)} \int_{1 / F_{0}(\omega)}^{1 / F_{t}(\omega)} f(\varrho \omega) \varrho^{n-1} d \varrho
\end{aligned}
$$

where $h_{t}(\omega)=\frac{1}{F_{t}(\omega)}-\frac{1}{F_{0}(\omega)}$ and we have applied (13). Now, we would like to pass to the limit when $t \rightarrow 0^{+}$by using the Lebesgue's dominated convergence theorem. Clearly, for a.e. $\omega \in S^{n-1}$, as $t \rightarrow 0^{+}, g_{t}(\omega)$ tends to

$$
\left(\frac{1}{F_{0}(\omega)}\right)^{2} F_{0}(\omega) P_{\Omega}\left(\nabla F_{0}(\omega)\right) f\left(\frac{\omega}{F_{0}(\omega)}\right)\left(\frac{1}{F_{0}(\omega)}\right)^{n-1}=P_{\Omega}\left(\nabla F_{0}(\omega)\right) f\left(\frac{\omega}{F_{0}(\omega)}\right)\left(\frac{1}{F_{0}(\omega)}\right)^{n} .
$$

We have to say that for the factor $\int_{0}^{1} F_{s t}(\omega) P_{\Omega}\left(\nabla F_{s t}(\omega)\right) d s$ the convergence holds may be only for a sequence $t_{j} \rightarrow 0^{+}$because of the continuity of $t \mapsto \nabla F_{t}$ with values in $L^{1}\left(S^{n-1}\right)$, but this does not matter. As for the domination condition, we can check it as follows :

$$
\left|g_{t}(\omega)\right| \leq \frac{1}{t}\left|\frac{1}{F_{t}(\omega)}-\frac{1}{F_{0}(\omega)}\right| \sup _{\frac{1}{F_{0}(\omega)} \leq \varrho \leq \frac{1}{F_{t}(\omega)}}\left|f(\varrho \omega) \varrho^{n-1}\right| \leq \frac{\left|F_{t}(\omega)-F_{0}(\omega)\right|}{t F_{0}(\omega) F_{t}(\omega)} \sup _{\alpha \leq \varrho \leq \beta}\left|f(\varrho \omega) \varrho^{n-1}\right|,
$$


where $\alpha$ and $\beta$ are positive constants. In fact, we have $B(0, r) \subset \Omega_{t} \subset D$ for all $t \in[0,1]$, so that $\frac{1}{F_{B(0, r)}} \leq \frac{1}{F_{t}} \leq \frac{1}{F_{D}}$, and we can take $\alpha=\inf _{S^{n-1}}\left(1 / F_{B(0, r)}\right)$ and $\beta=\sup _{S^{n-1}}\left(1 / F_{D}\right)$. Now, if we pass to the limit when $k \rightarrow \infty$ in (17), we get

$$
\left|F_{t}(\omega)-F_{0}(\omega)\right| \leq \frac{t}{r^{2}} \sup _{S^{n-1}} P_{D}, \quad t \in[0,1] .
$$

Hence,

$$
\left|g_{t}(\omega)\right| \leq \frac{\beta^{2}}{r^{2}} \sup _{S^{n-1}} P_{D} \sup _{\alpha \leq \varrho \leq \beta}\left|f(\varrho \omega) \varrho^{n-1}\right| .
$$

It remains just to apply the following (well known) lemma whose proof is left to the reader :

Lemma 5 For all $\varphi \in W_{l o c}^{1,1}(\mathbb{R})$ and all bounded intervals $I \subset \mathbb{R}$, we have

$$
\|\varphi\|_{L^{\infty}(I)} \leq \frac{1}{|I|} \int_{I}|\varphi(t)| d t+\int_{I}\left|\varphi^{\prime}(t)\right| d t
$$

where $|I|$ is the length of $I$.

The application of this lemma yields

$$
\left|g_{t}(\omega)\right| \leq C \frac{\beta^{2}}{r^{2}} \sup _{S^{n-1}} P_{D} \int_{\alpha}^{\beta}(|f(\varrho \omega)|+|\nabla f(\varrho \omega)|) \varrho^{n-1} d \varrho,
$$

where the constant $C$ only depends on $(\alpha, \beta)$. Since $f \in W_{l o c}^{1,1}\left(\mathbb{R}^{n}\right)$, it follows from Fubini's theorem that the function $\omega \mapsto \int_{\alpha}^{\beta}(|f(\varrho \omega)|+|\nabla f(\varrho \omega)|) \varrho^{n-1} d \varrho$ is in $L^{1}\left(S^{n-1}\right)$. Therefore, by Lebesgue's theorem, we can conclude that

$$
\lim _{t \rightarrow 0^{+}} I_{t}=\int_{S^{n-1}} P_{\Omega}\left(\nabla F_{0}(\omega)\right) f\left(\frac{\omega}{F_{0}(\omega)}\right) \frac{1}{F_{0}(\omega)^{n}} d \omega .
$$

The last step in the proof of Theorem 1 is to perform a change of variables to transform the integral on $S^{n-1}$ into an integral on $\partial \Omega_{0}$. Consider the map $\Phi$ defined by $\Phi(0)=0$ and

$$
\Phi(x)=\frac{F_{0}(x)}{|x|} x, \quad x \in \mathbb{R}^{n}, x \neq 0 .
$$

It is easy to check that it is a bi-Lipschitz homeomorphism from $\mathbb{R}^{n}$ onto itself whose inverse $\Psi$ is defined by $\Psi(0)=0$ and

$$
\Psi(y)=\frac{|y|}{F_{0}(y)} y, \quad y \in \mathbb{R}^{n}, y \neq 0
$$

It is also clear that $\Phi\left(\Omega_{0}\right)=B(0,1)$ and $\Phi\left(\partial \Omega_{0}\right)=S^{n-1}$. Let us denote by $I_{0}$ the integral given by (24) and let us perform the change of variables $\omega=\Phi(x)$ in it. See for example [7] or [12] for Lipschitz changes of variables. We get

$$
I_{0}=\int_{\partial \Omega_{0}} P_{\Omega}\left(\nabla F_{0}(x)\right) f(x) \frac{|x|^{n}}{F_{0}(x)^{2 n}} \operatorname{Jac}_{T}(\Phi)(x) d \sigma(x),
$$


where $\operatorname{Jac}_{T}(\Phi)$ is what is called the tangential Jacobian of $\Phi$. It is defined by

$$
\operatorname{Jac}_{T}(\Phi)(x)=\left|{ }^{t} \Phi^{\prime}(x)^{-1} \nu_{0}(x)\right|\left|\operatorname{det}\left(\Phi^{\prime}(x)\right)\right| .
$$

Recall that $\nu_{0}(x)$ is the exterior unit normal vector to $\partial \Omega_{0}$ at $x$ and that it is given a.e. on $\partial \Omega_{0}$ by $\nabla F_{0}(x) /\left|\nabla F_{0}(x)\right|$. Now, let us compute this tangential Jacobian. First, note that $\Phi^{\prime}(x)^{-1}=\Psi^{\prime}(y)$ with $y=\Phi(x)$; so, we have

$$
{ }^{t} \Phi^{\prime}(x)^{-1}={ }^{t} \Psi^{\prime}(y)=\frac{|y|}{F_{0}(y)} I d+\frac{1}{|y| F_{0}(y)} y^{t} y-\frac{|y|}{F_{0}(y)^{2}} \nabla F_{0}(y)^{t} y
$$

and

$$
\begin{aligned}
{ }^{t} \Phi^{\prime}(x)^{-1} \nu_{0}(x) & =\frac{|y|}{F_{0}(y)} \nu_{0}(x)+\frac{1}{|y| F_{0}(y)} y^{t} y \nu_{0}(x)-\frac{|y|}{F_{0}(y)^{2}} \nabla F_{0}(y)^{t} y \nu_{0}(x) \\
& =\frac{|x|}{F_{0}(x)} \nu_{0}(x)+\frac{1}{|x| F_{0}(x)} x^{t} x \nu_{0}(x)-\frac{|x|}{F_{0}(x)^{2}} \nabla F_{0}(x)^{t} x \nu_{0}(x) \\
& =\frac{|x|}{F_{0}(x)} \nu_{0}(x)+\frac{1}{|x|\left|\nabla F_{0}(x)\right|} x-\frac{|x|}{F_{0}(x)} \nu_{0}(x)=\frac{1}{|x|\left|\nabla F_{0}(x)\right|} x
\end{aligned}
$$

since ${ }^{t} x \nu_{0}(x)={ }^{t} x \nabla F_{0}(x) /\left|\nabla F_{0}(x)\right|=F_{0}(x) /\left|\nabla F_{0}(x)\right|$, thanks to Euler's relation. Hence,

$$
\left|{ }^{t} \Phi^{\prime}(x)^{-1} \nu_{0}(x)\right|=\frac{1}{\left|\nabla F_{0}(x)\right|} .
$$

On the other hand, we have

$$
\begin{aligned}
\operatorname{det}\left(\Phi^{\prime}(x)\right) & =\operatorname{det}\left(\frac{F_{0}(x)}{|x|} I d-\frac{F_{0}(x)}{|x|^{3}} x^{t} x+\frac{1}{|x|} x^{t} \nabla F_{0}(x)\right) \\
& =\left(\frac{F_{0}(x)}{|x|}\right)^{n} \operatorname{det}\left(I d-\frac{x^{t} x}{|x|^{2}}+\frac{x^{t} \nabla F_{0}(x)}{F_{0}(x)}\right)=\left(\frac{F_{0}(x)}{|x|}\right)^{n} \operatorname{det}\left(I d+x^{t} z\right)
\end{aligned}
$$

where $z=-\frac{x}{|x|^{2}}+\frac{\nabla F_{0}(x)}{F_{0}(x)}$. Applying the more or less known formula

$$
\operatorname{det}\left(I d+x^{t} z\right)=1+{ }^{t} x z
$$

we get

$$
\operatorname{det}\left(\Phi^{\prime}(x)\right)=\left(\frac{F_{0}(x)}{|x|}\right)^{n}\left(1-\frac{{ }^{t} x x}{|x|^{2}}+\frac{{ }^{t} x \nabla F_{0}(x)}{F_{0}(x)}\right)=\left(\frac{F_{0}(x)}{|x|}\right)^{n}
$$

Hence,

$$
\operatorname{Jac}_{T}(\Phi)(x)=\frac{F_{0}(x)^{n}}{\left|\nabla F_{0}(x)\right||x|^{n}}
$$

Finally,

$$
I_{0}=\int_{\partial \Omega_{0}} P_{\Omega}\left(\nabla F_{0}(x)\right) f(x) \frac{|x|^{n}}{F_{0}(x)^{2 n}} \frac{F_{0}(x)^{n}}{\left|\nabla F_{0}(x)\right||x|^{n}} d \sigma(x)=\int_{\partial \Omega_{0}} P_{\Omega}\left(\nu_{0}(x)\right) f(x) d \sigma(x),
$$

since $F_{0}=1$ on $\partial \Omega_{0}$, and this achieves the proof of Theorem 1 . 


\section{Appendix}

Proof of Lemma 3 : We shall use the Fourier transformation $\mathscr{F}$ and denote by $\xi$ the variable which is dual to $x$, that is, in the Fourier side. We use both notations $\mathscr{F}(T)$ and $\widehat{T}$ for the Fourier transform of $T$.

Let us first remark that, by density of $\mathscr{D}\left(\mathbb{R}^{n}\right)$ in $\mathscr{S}\left(\mathbb{R}^{n}\right)$, the estimates satisfied by $T$ and $\partial_{i} \partial_{j} T$ hold also for $\varphi \in \mathscr{S}\left(\mathbb{R}^{n}\right)$. If $\lambda$ is an arbitrary positive number, we can write, using the Parseval-Plancherel formula,

$$
\begin{aligned}
\left\langle\partial_{j} T, \varphi\right\rangle & =(2 \pi)^{-n}\left\langle i \xi_{j} \widehat{T}, \widehat{\varphi}(-\xi)\right\rangle=(2 \pi)^{-n}\left\langle\left(\lambda+\lambda^{-1} \xi^{2}\right) \widehat{T},\left(\lambda+\lambda^{-1} \xi^{2}\right)^{-1} i \xi_{j} \widehat{\varphi}(-\xi)\right\rangle \\
& =-\left\langle\left(\lambda-\lambda^{-1} \Delta\right) T,\left(\lambda-\lambda^{-1} \Delta\right)^{-1} \partial_{j} \varphi\right\rangle=-\lambda\left\langle T, P_{\lambda}(\varphi)\right\rangle+\lambda^{-1}\left\langle\Delta T, P_{\lambda}(\varphi)\right\rangle
\end{aligned}
$$

where $\Delta$ is the Laplace operator and $P_{\lambda}=\left(\lambda-\lambda^{-1} \Delta\right)^{-1} \partial_{j}$. Then, it follows from the assumptions that, for all $\varphi \in \mathscr{D}\left(\mathbb{R}^{n}\right)$,

$$
\left|\left\langle\partial_{j} T, \varphi\right\rangle\right| \leq\left(\lambda C_{1}+\lambda^{-1} n C_{2}\right)\left\|P_{\lambda}(\varphi)\right\|_{L^{\infty}} .
$$

Assume for a moment that the operator $P_{\lambda}$ satisfies the inequality

$$
\left\|P_{\lambda}(\varphi)\right\|_{L^{\infty}} \leq C_{3}\|\varphi\|_{L^{\infty}}
$$

for all $\varphi \in \mathscr{D}\left(\mathbb{R}^{n}\right)$, where $C_{3}$ is a constant that depends only on the dimension $n$. We can then finish the proof as follows. For all $\varphi \in \mathscr{Q}\left(\mathbb{R}^{n}\right)$ and all $\lambda>0$, we have the inequality

$$
\left|\left\langle\partial_{j} T, \varphi\right\rangle\right| \leq\left(\lambda C_{1}+\lambda^{-1} n C_{2}\right) C_{3}\|\varphi\|_{L^{\infty}} .
$$

Now, by taking the infimum on $\lambda>0$, we obtain

$$
\left|\left\langle\partial_{j} T, \varphi\right\rangle\right| \leq 2 C_{3} \sqrt{n C_{1} C_{2}}\|\varphi\|_{L^{\infty}}=C \sqrt{C_{1} C_{2}}\|\varphi\|_{L^{\infty}} .
$$

It remains to show (26). In fact, we have

$$
\mathscr{F}\left(P_{\lambda}(\varphi)\right)(\xi)=\widehat{G}\left(\lambda^{-1} \xi\right) \widehat{\varphi}(\xi) \quad \text { with } \quad \widehat{G}(\xi)=\frac{i \xi_{j}}{1+\xi^{2}}
$$

Since (see for example [10], page 132)

$$
\mathscr{F}^{-1}\left(\frac{1}{1+\xi^{2}}\right)=\frac{1}{4 \pi} \int_{0}^{\infty} \mathrm{e}^{-\pi x^{2} / t} \mathrm{e}^{-t / 4 \pi} t^{-n / 2} d t
$$

we have in fact

$$
G(x)=\frac{1}{4 \pi} \partial_{x_{j}} \int_{0}^{\infty} \mathrm{e}^{-\pi x^{2} / t} \mathrm{e}^{-t / 4 \pi} t^{-n / 2} d t=\frac{1}{4 \pi} \int_{0}^{\infty} \mathrm{e}^{-\pi x^{2} / t}\left(-\frac{2 \pi x_{j}}{t}\right) \mathrm{e}^{-t / 4 \pi} t^{-n / 2} d t .
$$

Now, it is easy to see that $G \in L^{1}\left(\mathbb{R}^{n}\right)$, and since we can write

$$
P_{\lambda}(\varphi)=G_{\lambda} \star \varphi \quad \text { with } \quad G_{\lambda}(x)=\lambda^{n} G(\lambda x),
$$

it follows from young's inequality that

$$
\left\|P_{\lambda}(\varphi)\right\|_{L^{\infty}} \leq\left\|G_{\lambda}\right\|_{L^{1}}\|\varphi\|_{L^{\infty}}=\|G\|_{L^{1}}\|\varphi\|_{L^{\infty}}=C_{3}\|\varphi\|_{L^{\infty}},
$$

which achieves the proof of Lemma 3 . 


\section{References}

[1] Niftiyev, A.A.; Gasimov Y.S. Control by boundaries and eigenvalue problems with variable domains, Publishing House of Baku State University, (in Russian), 2004.

[2] Boulkhemair, A.; Chakib, A., On a shape derivative formula with respect to convex domains, Journal of Convex Analysis, Volume 21 (2014), No. 1, 67-87.

[3] Boulkhemair, A.; Chakib, A., Erratum : On a shape derivative formula with respect to convex domains, submitted to Journal of Convex Analysis.

[4] Demyanov, V.P.; Rubinov, A.M., Bases of non-smooth analysis and quasi-differential calculus, Nauka, Moscow, (in Russian), 1990.

[5] Henrot, A.; Pierre, M., Variation et optimisation de formes. Une analyse géométrique, Mathematics and Applications, 48, Springer, Berlin, 2005.

[6] Hörmander, L., Notions of convexity, Progress in Mathematics, 127. Birkhäuser Boston, Inc., Boston, MA, 1994.

[7] Evans, L.; Gariepy, R., Measure theory and fine properties of functions, CRC Press, 1992.

[8] Webster, L., Convexity, Oxford Science Publications, 1994.

[9] Schneider, R., Convex bodies : The Brunn-Minkowski theory, Cambridge University Press, 1993.

[10] Stein, E. M., Singular integrals and differentiability properties of functions, Princeton University Press, 1970.

[11] Burago, Y.D., Zalgaller, V.A., Geometric inequalities, Transl. by A.B. Sossinsky, Springer-Verlag, 1988.

[12] Droniou, J., Quelques Résultats sur les Espaces de Sobolev, Polycopié de l'Ecole Doctorale de Maths-Info de Marseille, (in french), 2001, available at http://users.monash.edu.au/ jdroniou/travaux-en.html\#publisautres 